\title{
Elevated expression level of long noncoding RNA MALAT-1 facilitates cell growth, migration and invasion in pancreatic cancer
}

\author{
FENG JIAO $^{1 *}$, HAI HU ${ }^{1 *}$, CUNCUN YUAN ${ }^{2}$, LEI WANG ${ }^{1}$, WEIHUA JIANG ${ }^{1}$, \\ ZILIANG JIN $^{1}$, ZHEN GUO ${ }^{1}$ and LIWEI WANG ${ }^{1}$ \\ ${ }^{1}$ Department of Oncology and Shanghai Key Laboratory of Pancreatic Diseases; ${ }^{2}$ Department of Pathology, \\ First People's Hospital, School of Medicine, Shanghai Jiao Tong University, Shanghai 201620, P.R. China
}

Received June 13, 2014; Accepted September 4, 2014

DOI: $10.3892 / o r .2014 .3518$

\begin{abstract}
Pancreatic cancer is one of the most aggressive solid malignancies with a dismal survival rate. Recent studies have shown that high expression levels of long noncoding RNA (lncRNA) metastasis-associated lung adenocarcinoma transcript-1 (MALAT-1) correlate with several solid tumors. However, the underlying molecular mechanisms and its clinical significance in pancreatic cancer remain to be elucidated. In the present study, our results showed that MALAT-1 expression levels were upregulated in pancreatic cancer tissues compared with adjacent noncancerous controls. Consistently, higher expression level of MALAT-1 was found in all seven pancreatic cancer cell lines relative to the human pancreatic ductal epithelial cell. Further function analysis revealed that downregulation of MALAT-1 could inhibit tumor cell proliferation and decrease cell migration and invasion in vitro. The underlying mechanisms are possibly involved in inducing $\mathrm{G}_{2} / \mathrm{M}$ cell cycle arrest, promoting cell apoptosis, suppressing epithelial-mesenchymal transition and reducing cancer stem-like properties. In conclusion, this study indicated that MALAT-1 may serve as an oncogenic lncRNA that is involved in malignancy phenotypes of pancreatic cancer. Therefore, it may be used as a potential therapeutic target.
\end{abstract}

\section{Introduction}

Pancreatic cancer is an aggressive disease with a 5-year survival rate of less than $5 \%$ and a median survival of 6 months

Correspondence to: Dr Lei Wang or Professor Liwei Wang, Department of Oncology and Shanghai Key Laboratory of Pancreatic Diseases, First People's Hospital, School of Medicine, Shanghai Jiao Tong University, 650 New Songjiang Road, Shanghai 201620, P.R. China

E-mail: wang_lei744@hotmail.com

E-mail: yzwlw64@gmail.com

*Contributed equally

Key words: pancreatic cancer, epithelial-mesenchymal transition, metastasis-associated lung adenocarcinoma transcript-1, invasion, long noncoding RNA after diagnosis, thereby exhibiting the poorest prognosis of all solid tumors (1). It is characterized by a high propensity for local invasion and distant metastasis as well as a largely drugresistant phenotype; however, the molecular events underlying this remain to be elucidated.

Recent advances in genome analysis, including microarray and massively parallel sequencing, have discovered extensive transcription of large RNA transcripts that lack coding protein function, termed long noncoding RNAs (lncRNAs). It is becoming evident that IncRNAs may be an important class of pervasive genes involved in tumorigenesis and metastasis (2). Metastasis-associated lung adenocarcinoma transcript-1 (MALAT-1) is an evolutionarily highly conserved and ubiquitously expressed IncRNA with a length of 8,700 nucleotides (3). Since its discovery in non-small cell lung cancer (3), MALAT-1 has been linked to several human tumor entities. In most cases, MALAT-1 is expressed higher in tumor tissues; it may be closely related to clinical parameters and may control tumor cell progression (4). However, little is known about the expression pattern and biological function of MALAT-1 involved in pancreatic cancer.

In the present study, we demonstrated that MALAT-1 expression levels were upregulated in pancreatic cancer tissue compared with adjacent noncancerous controls. Consistently, higher expression level of MALAT-1 was found in all seven pancreatic cancer cell lines relative to the human pancreatic ductal epithelial (HPDE) cells. Furthermore, our data revealed that knockdown of MALAT-1 inhibited tumor cell growth via induction of $\mathrm{G}_{2} / \mathrm{M}$ cell cycle arrest and apoptosis, and decreased cell migration and invasion through regulation of epithelial-mesenchymal transition (EMT) and stem-like cell marker expression.

\section{Materials and methods}

Human tissue samples and cell lines. Human tumor tissue samples and adjacent noncancerous controls were obtained by surgical resection from six patients with pancreatic cancer, at the Department of General Surgery, First People's Hospital, School of Medicine, Shanghai Jiao Tong University, Shanghai, China. All samples were derived from patients who had not received adjuvant treatment including radiotherapy or chemotherapy prior to surgery. All samples were snap-frozen and 
stored in liquid nitrogen after collection. Written informed consent was obtained from all subjects, and the study was approved and supervised by the Ethics Committee of the First People's Hospital, School of Medicine, Shanghai Jiao Tong University.

HPDE, pancreatic cancer cells BxPC-3, CFPAC-1, CAPAN-1, SW1990, AsPC-1, PANC-1 and HS-766T were all obtained from Chinese Academy of Sciences Cell Bank (Shanghai, China). PANC-1 and HS-766T were grown in $5 \% \mathrm{CO}_{2}$ saturated humidity, at $37^{\circ} \mathrm{C}$, and cultured in DMEM supplemented with $2 \mathrm{mmol} / \mathrm{l}$ glutamine and $10 \%$ fetal bovine serum (FBS) (both from Gibco, USA) and subcultured by harvesting with trypsin-EDTA. HPDE, BxPC-3, CAPAN-1, AsPC-1, CFPAC-1 and SW1990 cells were cultured in RPMI-1640 (Gibco) supplemented with 10\% FBS.

Real-time-quantitative polymerase chain reaction ( $R T-q P C R)$ analysis. Total RNA was isolated from the cultured cells and tissue samples by using an RNA isolation kit (Takara Bio, Inc.) according to the manufacturer's instructions. Reverse transcription and RT-qPCR kits (Takara Bio, Inc.) were applied to evaluate expression of MALAT-1. Primers used were: MALAT-1 forward, 5'-GAATTGCGTCATTTAAAGCCTA GTT-3' and reverse, 5'-GTTTCATCCTACCACTCCCAAT TAAT-3'; GAPDH forward, 5'-ACAGTCAGCCGCATCTT CTT-3' and reverse, 5'-GACAAGCTTCCCGTTCTCAG-3'. The expression of GAPDH was detected as the endogenous control. Relative mRNA expression of MALAT-1 was calculated with the comparative threshold cycle $(\mathrm{Ct})\left(2^{-\Delta \Delta \mathrm{Ct}}\right)$ method.

Western blot analysis. Cells were lysed in RIPA lysis buffer and the protein concentration was determined (Beyotime). Total proteins were fractionated using SDS-PAGE and transferred onto a polyvinylidene fluoride membrane. The membranes were blocked in 3\% bovine serum albumin in TBST buffer containing $0.1 \%$ Tween-20 and then incubated with the indicated primary antibodies at $4^{\circ} \mathrm{C}$ overnight. Appropriate secondary antibodies were incubated at room temperature for $1 \mathrm{~h}$ and detected using the enhanced chemiluminescence detection system. The data was adjusted against loading control using $\beta$-actin. The antibodies used for western blot analyses were: mouse anti-Ncadherin, mouse anti-E-cadherin (BD Biosciences, Bedford, MA, USA); mouse anti-vimentin, rabbit anti-ALDH (Abcam); rabbit anti-Slug, rabbit anti-Snail, mouse anti-CDC2, rabbit anti-matrix metalloproteinases-2 (MMP-2), rabbit anti-MMP-9, rabbit anti-PCNA (Cell Signaling Technology); mouse anti-p21, mouse anti-p53, mouse anti-CD44, rabbit anti-CD24, mouse anti- $\beta$-actin (Santa Cruz Biotechnology, Inc., Santa Cruz, CA, USA).

Establishment of pancreatic cancer cell line with stable expression of MALAT-1 short hairpin RNA (shRNA). Based on principles of shRNA design and the human MALAT-1 structure (NR_002819), three plasmid vectors encoding shRNA directed against MALAT-1 mRNA were constructed. The three shRNA targeting sequences were: shRNA-M1, 5'-GAG TAACTGGCATGTGAGCAA-3'; shRNA-M2, 5'-CATGACG GAGGTTGAGATGAA-3'; shRNA-M3, 5'-AAGCCGAA ATAAATGAGAGAT-3'. The scrambled sequence, 5'-TTC TCCGAACGTGTCACGT-3', was used as a negative control that does not target any known human mRNA. The preparation of lentivirus expressing human MALAT-1 shRNA was performed using the GV-248 lentiviral RNAi expression system (Genechem, Shanghai, China). AsPC-1 and CFPAC-1 were infected with lentiviral particles containing specific or negative control vectors, and the polyclonal cells with puromycin resistance were selected for further experiments.

Colony formation assay. Briefly, $\sim 1,000$ cells were added to each well of a 6-well culture plate. After 2 weeks of incubation, cell colonies were washed twice with PBS, fixed with $4 \%$ paraformaldehyde for $15 \mathrm{~min}$ and then stained with crystal violet for $30 \mathrm{~min}$. Individual clones with $>50$ cells were counted.

Cell proliferation assay. A real-time imaging system (IncuCyte ${ }^{\mathrm{TM}}$ ) was used to measure cell proliferation using non-label cell monolayer confluence approach. IncuCyte provides the ability to acquire high quality, phase-contrast images and an integrated confluence metric as a surrogate for cell number (5). We used a similar approach to determine the effect of MALAT-1 on pancreatic cancer cell proliferation. Cell confluence was compared between M-nc and M-sil stable transfected cancer cells.

Wound healing assay. Cells were cultured and grown to $100 \%$ confluence. Wounds were scratched in the monolayer with a $100-\mu 1$ pipette tip. Suspended cells and debris were washed with PBS buffer, then the cells were incubated in RPMI-1640 medium containing $2 \% \mathrm{FBS}$ at $37^{\circ} \mathrm{C}$. The migration of cells into the wounded areas was evaluated at the indicated times using an inverted microscope, and then photographed. Three different areas in each assay were selected to measure the distance of the migrating cells to the origin of the wound.

Boyden chamber and Transwell assay. The cell invasive and migratory potential were evaluated using Boyden chamber and Transwell assay, respectively. Briefly, Boyden chamber was conducted using specialized MilliCell chambers, which included a 24-well tissue culture plate with 12 cell culture inserts (Millipore, Bedford, MA, USA). The inserts contained an $8 \mu \mathrm{m}$ pore size polycarbonate membrane with a precoated thin layer of Matrigel (BD Biosciences). Ten percent FBS-containing medium was placed in the lower chambers to act as a chemo-attractant. Then, $1 \times 10^{5}$ AsPC-1 and CFPAC-1 in a $100 \mu \mathrm{l}$ volume of serum-free medium were placed in the upper chambers and incubated at $37^{\circ} \mathrm{C}$ for $48 \mathrm{~h}$. Invasive cells on the lower surface of the membrane, which had invaded the Matrigel and had migrated through the polycarbonate membrane, were stained by the staining solution, and counted under a microscope in five randomly selected fields at a magnification of $x 200$. Transwell assay was the same as the Boyden chamber with the exception that no Matrigel was used and the permeating time for cells was $24 \mathrm{~h}$.

Flow cytometry analysis of cell apoptosis and cell cycle. For analysis of the cell apoptosis, cells were harvested at 70-80\% confluence and incubated with reagent containing Annexin V-FITC and propidium iodide (BD Biosciences) for $15 \mathrm{~min}$ in darkness at room temperature. Apoptotic cells were analyzed using FACSCalibur flow cytometer (BD Biosciences). 

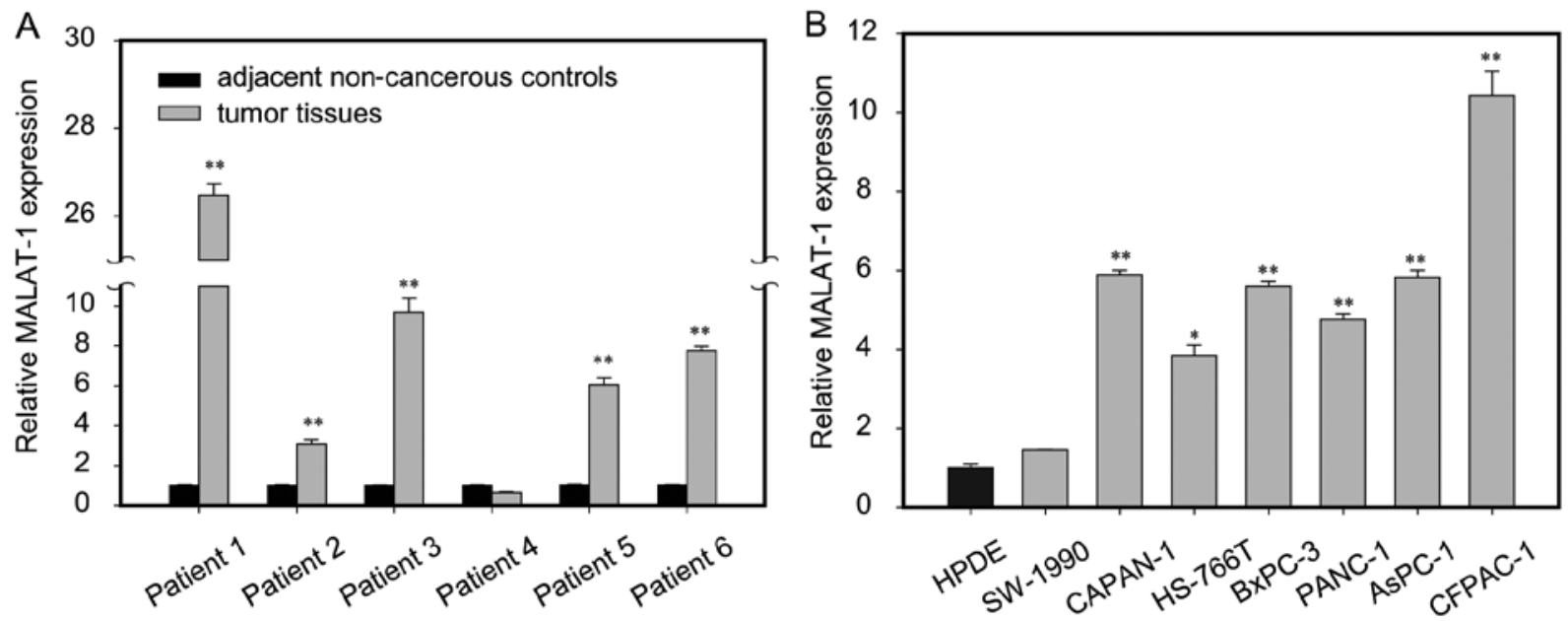

Figure 1. Higher levels of metastasis-associated lung adenocarcinoma transcript-1 (MALAT-1) expression in pancreatic cancer. Relative MALAT-1 concentration was determined using real-time-quantitative polymerase chain reaction (RT-qPCR). The analysis of MALAT-1 expression level was performed in (A) pancreatic cancer tissues $(\mathrm{n}=6)$ and adjacent non-cancerous pancreas tissues, as well as in (B) seven pancreatic cancer cell lines and human normal pancreatic ductal epithelial (HPDE) cells. Data are presented as mean $\pm \mathrm{SEM}$ for three independent experiments. ${ }^{*} \mathrm{P}<0.05$ and ${ }^{* *} \mathrm{P}<0.01$ compared with the respective control groups.

For cell cycle analysis, cells were fixed in $70 \%$ ethanol at $4^{\circ} \mathrm{C}$ overnight and then treated with RNase A $(50 \mu \mathrm{g} / \mathrm{ml})$ and stained with propidium iodide $(25 \mu \mathrm{g} / \mathrm{ml})$ for $30 \mathrm{~min}$ at $37^{\circ} \mathrm{C}$. Distribution of cell-cycle phases was determined using ModFit software (BD Biosciences).

Statistical analysis. All statistical analyses were performed using SPSS 13.0. The data are presented as means \pm SEM from at least three separate experiments. The difference between two groups was analyzed by the Student's t-test (two-tailed). In vitro cell growth assay was tested using factorial design one-way analysis of variance (ANOVA). The P-value of $<0.05$ was considered to indicate a statistically significant difference.

\section{Results}

MALAT-1 is upregulated in pancreatic cancer cell lines and tumor tissue samples. To assess the role of MALAT-1 in pancreatic cancer, we first assayed MALAT-1 expression in tumor tissue samples and adjacent non-cancerous pancreas tissues from six patients with pancreatic cancer. The results showed that MALAT-1 expression was significantly higher in five cases of tumor tissues than in matched paracancerous controls (Fig. 1A). Furthermore, we compared the expression of MALAT-1 between pancreatic cancer cell lines and normal HPDE cells. Notably, consistently higher expression level of MALAT-1 was found in all seven pancreatic cancer cell lines relative to the HPDE, among which five were upregulated $>4$-fold (Fig. 1B). Taken together, it is suggested that upregulation of MALAT-1 is a frequent event in human pancreatic cancer.

Stable downregulation of MALAT-1 expression inhibits cell proliferation in vitro. Among the pancreatic cancer cell lines, AsPC-1 and CFPAC-1 had relatively higher expression levels of MALAT-1 (Fig. 1B), therefore these two cell lines were chosen to study the functions of endogenous MALAT-1 through a loss-of-function approach. We used a lentiviral shRNA vector to specifically and stably knock down the expression of MALAT-1 in AsPC-1 and CFPAC-1. A knockdown effect was observed by RT-qPCR, and we found that M-sil achieved the greatest efficacy in silencing MALAT-1 expression compared to the negative control M-nc (Fig. 2A). Thus, stable transfection cancer cell lines AsPC-1/M-nc and AsPC-1/M-si1, as well as CFPAC-1/M-nc and CFPAC-1/M-si1 were used in the following experiments.

Subsequently, we examined the effect of decreased MALAT-1 expression on pancreatic cancer cell proliferation in vitro by the IncuCyte real-time video imaging system. The determined growth curves showed that suppressing MALAT-1 significantly reduced cell proliferation in comparison with M-nc cells (Fig. 2B). The analysis of plate clone formation assay in CFPAC-1 was concordant with the above result (Fig. 2C). However, for AsPC-1, the two groups had no cell clone formation. Furthermore, western blot analysis showed that, compared to M-nc, the level of proliferation marker PCNA was decreased in the M-sil groups (Fig. 2D).

Silencing of MALAT-1 induces $G_{2} / M$ cell cycle arrest and promotes cell apoptosis. Next, to investigate the mechanism involved in growth suppression, we carried out flow cytometry analysis. The cell cycle results showed that the $\mathrm{G}_{2} / \mathrm{M}$-phase fraction increased from $14.0 \pm 0.9 \%$ (AsPC-1/M-nc) to $23.9 \pm 0.7 \%$ (AsPC-1/M-si1), $1.8 \pm 0.2 \%$ (CFPAC-1/M-nc) to $4.6 \pm 0.7 \%$ (CFPAC-1/M-si1) respectively, indicating $\mathrm{G}_{2} / \mathrm{M}$ cell cycle arrest after MALAT-1 knockdown (Fig. 3A). In addition, we detected cell apoptosis after MALAT-1 downregulation. Compared to the M-nc, significantly enhanced cell apoptosis was observed in the M-sil group (AsPC-1/M-si1 6.46\% vs. AsPC-1/M-nc 3.45\%; CPFAC-1/M-si1 $15.59 \%$ vs. CPFAC-1/M-nc $5.55 \%$ ) (Fig. 3B).

The $G_{2}$ checkpoint prevents cells from entering mitosis when DNA is damaged, providing an opportunity for repair and stopping the proliferation of damaged cells. CDC2, 


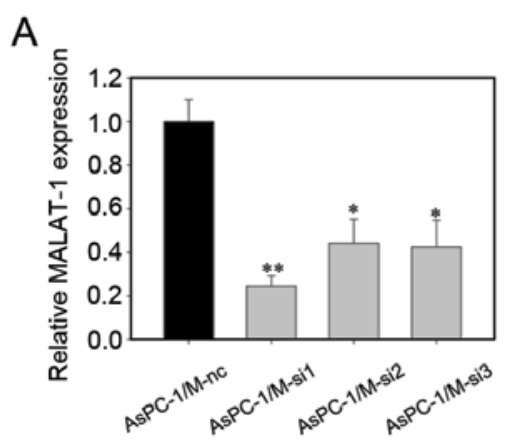

B

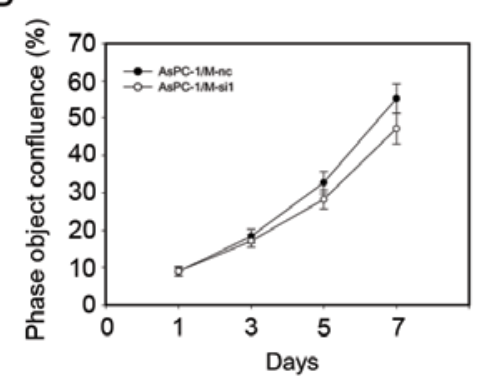

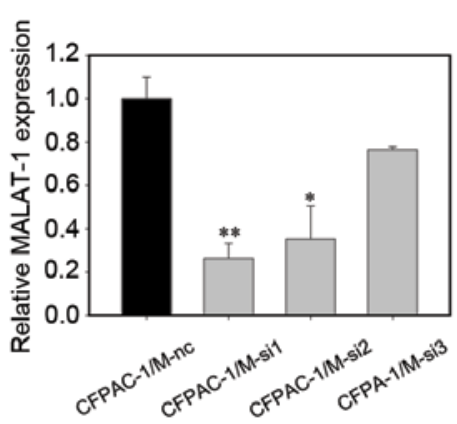

C
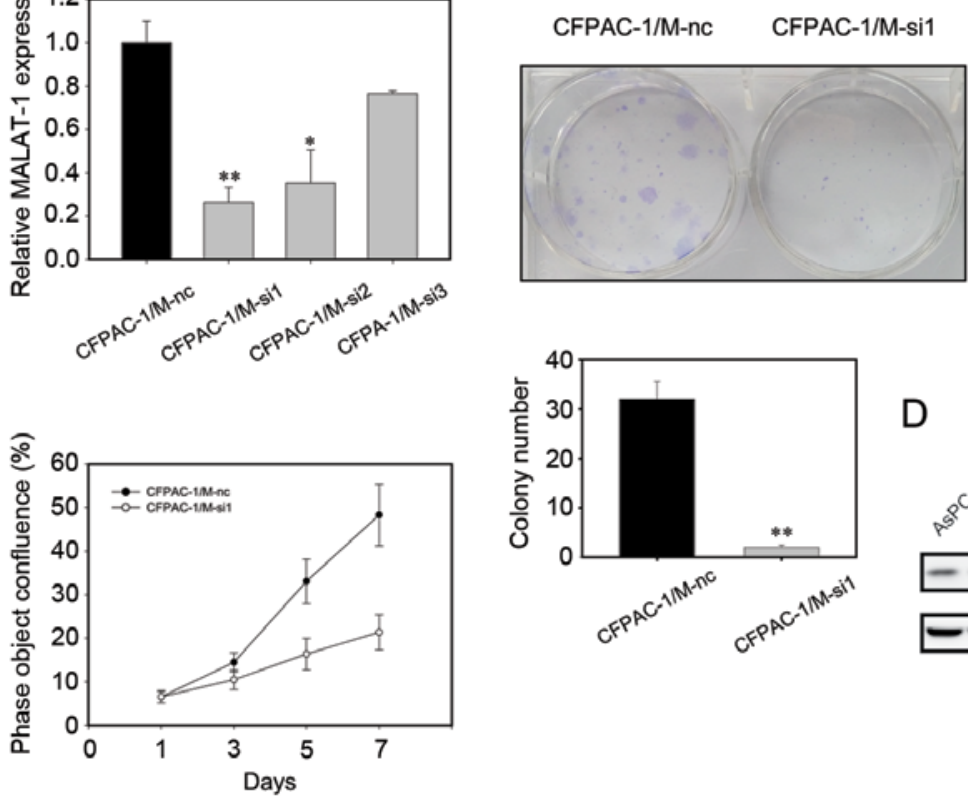

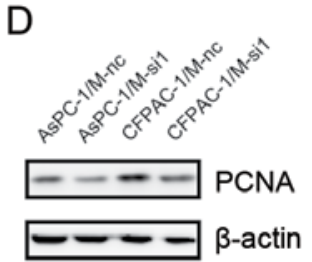

Figure 2. Stable downregulation of metastasis-associated lung adenocarcinoma transcript-1 (MALAT-1) expression inhibits cell growth in vitro. (A) Real-timequantitative polymerase chain reaction (RT-qPCR) confirmed the knockdown effect on MALAT-1 expression in AsPC-1 and CFPAC-1 cells. M-sil was chosen for subsequent experiments due to the greatest efficacy in silencing endogenous MALAT-1 expression. (B) IncuCyte ${ }^{\mathrm{TM}}$ was used to measure cell proliferation through non-label cell monolayer confluence approach. (C) Quantitative analysis of colony formation number. (D) Stable knockdown of MALAT-1 decreased the expression of proliferation marker PCNA in M-sil compared to M-nc groups by western blotting. Data are representative of at least three separate experiments. ${ }^{*} \mathrm{P}<0.05$ and ${ }^{* *} \mathrm{P}<0.01$ compared with the respective control groups.

the cyclin-dependent kinase that normally drives cells into mitosis, is the ultimate target of pathways that mediate rapid arrest in $\mathrm{G}_{2} / \mathrm{M}(6)$. A previous study revealed that $\mathrm{p} 53$ plays an important role in maintaining $\mathrm{G}_{2} / \mathrm{M}$ arrest and induces cell apoptosis (7). The contribution of p53 to $\mathrm{G}_{2} / \mathrm{M}$ arrest and cell apoptosis involves some of its transcriptional targets. p21, a well-known cell cycle regulator, is a major target of p53 (7). Elevated expression of p21 may result in increased association with CDKs and inhibit their activity, blocking the entry of cells at the $\mathrm{G}_{2}$-M-phase transition checkpoint, and induce apoptosis (8). Our results revealed that p21 and p53 protein levels significantly increased following MALAT-1 downregulation, while CDC2 expression decreased (Fig. 3C). Taken together, these results indicated an inhibiting effect of reduced MALAT-1 expression on pancreatic cancer cell growth possibly through inducing $\mathrm{G}_{2} / \mathrm{M}$ cell cycle arrest and promoting cell apoptosis.

Knockdown of MALAT-1 suppresses cell migration and invasion in vitro. To examine the effect of MALAT-1 on pancreatic cancer cell migration, we used a wound-healing assay and a Transwell migration assay. According to the wound-healing assay, the migratory areas of AsPC-1/M-si1 and CFPAC-1/M-si1 were significantly smaller than those of control cells (Fig. 4A). Similarly, Transwell migration assay showed that, after 24-h incubation, the number of migrated cells in the M-sil groups was significantly lower than that in the M-nc cells (AsPC-1/M-si1 45 \pm 4 vs. AsPC-1/M-nc

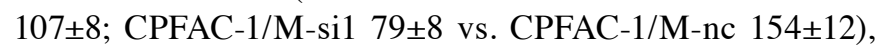
indicating a decreased migratory ability following MALAT-1 downregulation (Fig. 4B). Next, using a Boyden chamber pre-coated with Matrigel, we determined changes in cell invasiveness after $48 \mathrm{~h}$ incubation. Compared with the M-nc cells, M-si1 cells revealed significantly decreased invasiveness (AsPC-1/M-si1 27 \pm 2 vs. AsPC-1/M-nc 82 \pm ; CPFAC-1/M-si1 $18 \pm 2$ vs. CPFAC-1/M-nc 90 \pm 3 ) (Fig. 4C). Based on these results, knockdown of MALAT-1 clearly inhibited the migration and invasion of pancreatic cancer cells in vitro.

MALAT-1 facilitates tumor progression by inducing EMT. It is believed that acquiring the migratory characteristics of a mesenchymal-like state enhances the invasive capabilities of cancer cells. The EMT switch is related to an unfavorable prognosis in pancreatic ductal adenocarcinoma (9). During EMT of in situ tumor cells, expression of proteins that promote cell-cell contact such as E-cadherin can be lost, and mesenchymal markers such as vimentin, $\mathrm{N}$-cadherin and the metalloproteinases MMP-2 and MMP-9 can be acquired, resulting in enhanced ability for cell migration and invasion (10). We therefore examined the expression of EMT-related genes in response to the knockdown of MALAT-1. The results showed that the level of E-cadherin was upregulated with the decrease in the level of MALAT-1, while $\mathrm{N}$-cadherin and vimentin decreased (Fig. 5A). In addition, EMT-related transcriptional factors including Snail and Slug expression were also reduced (Fig. 5A). Moreover, we found that suppressing MALAT-1 expression decreased expression of MMP-2 and MMP-9 (Fig. 5A). Furthermore, CFPAC-1 cells were morphologically mixed populations of epithelial and spindle-shaped mesenchymal type cells in nature and led 


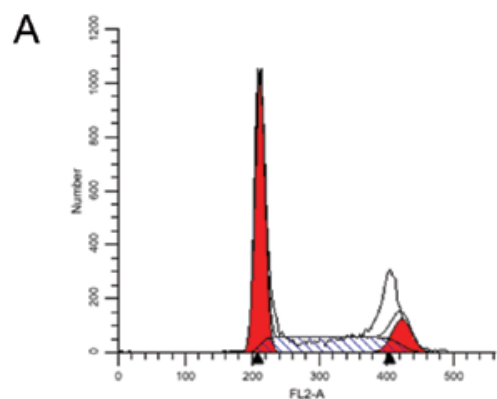

AsPC-1/M-nc

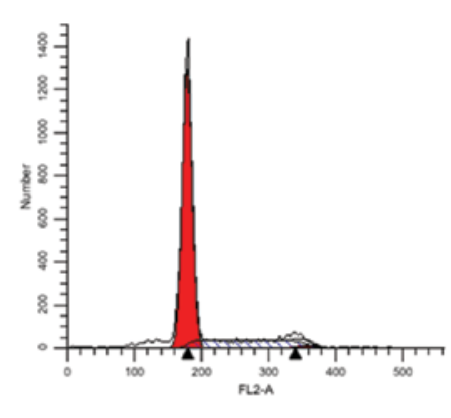

CFPAC-1/M-nc

B

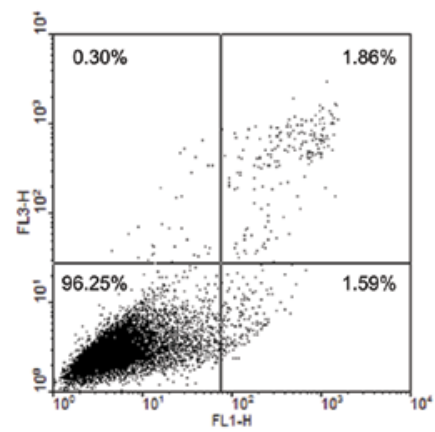

AsPC-1/M-nc

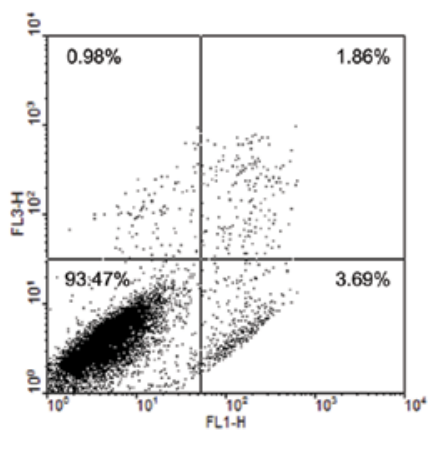

CFPAC-1/M-nc

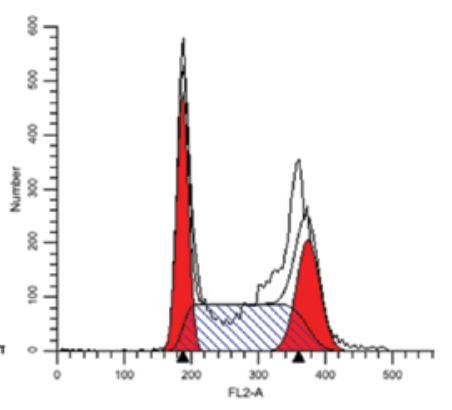

AsPC-1/M-si1

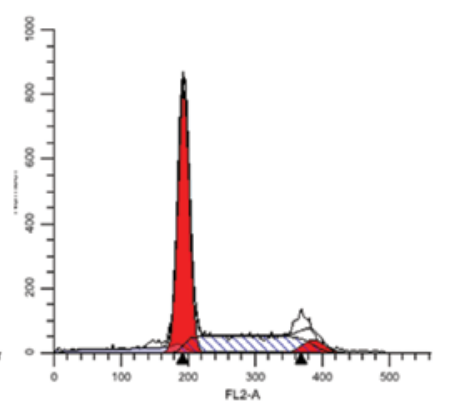

CFPAC-1/M-si 1

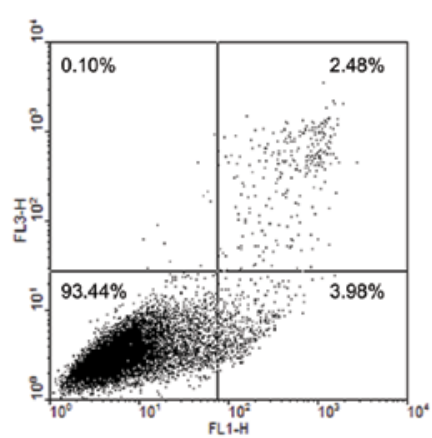

AsPC-1/M-si1

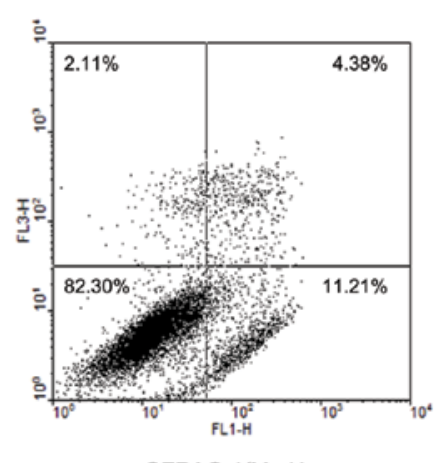

CFPAC-1/M-si1
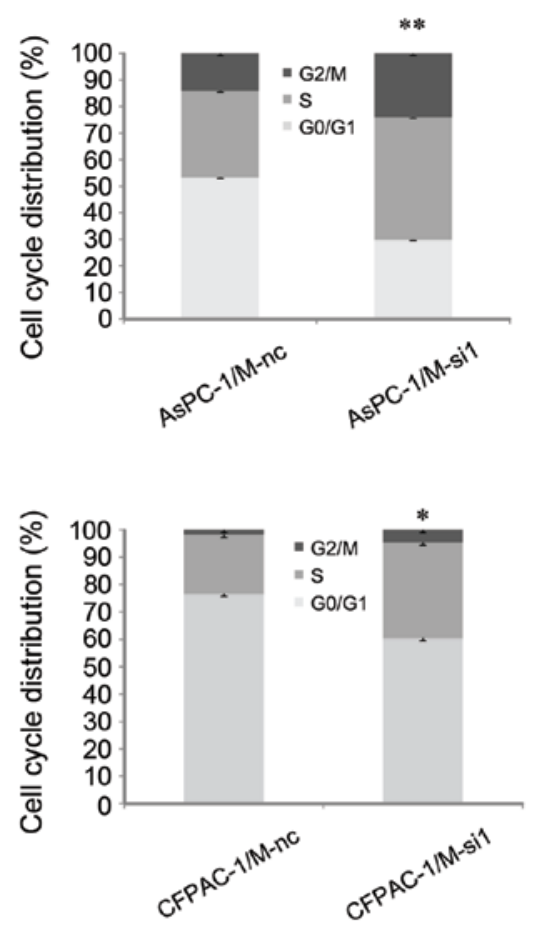

C

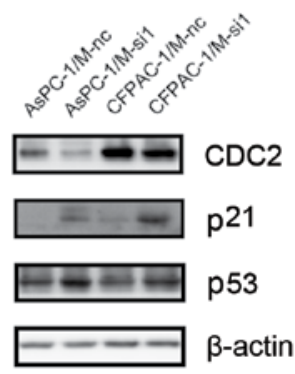

Figure 3. Silencing of metastasis-associated lung adenocarcinoma transcript-1 (MALAT-1) induces $\mathrm{G}_{2} / \mathrm{M}_{\text {cell }}$ cycle arrest and promotes cell apoptosis (A) Representative cell cycle images of flow cytometry analysis. Downregulation of MALAT-1 induced the cell cycle arrest at the $\mathrm{G}_{2} / \mathrm{M}_{\mathrm{H}} \mathrm{phase}$. (B) In comparisons with M-nc, cell apoptosis induction was observed in M-sil groups. (C) The protein levels of p53 and p21 were increased by knockdown of MALAT-1, while CDC2 expression decreased. Data are representative of at least three separate experiments. ${ }^{*} \mathrm{P}<0.05$ and ${ }^{* * *} \mathrm{P}<0.01$ compared with the respective control groups.

to typical epithelial morphology transition after MALAT-1 downregulation (Fig. 5B). However, the change of morphological features for MALAT-1 knockdown in AsPC-1 was not observed. Collectively, these findings suggest that knockdown of MALAT-1 inhibits pancreatic cancer cell migration and invasion possibly through suppression of EMT.
MALAT-1 knockdown decreases the expression of cancer stem-like cell markers. Accumulating evidence suggests that cells can acquire stem-like properties during induction of EMT (11). Pancreatic cancer stem cells (CSCs) have been identified based on the expression of CD24, CD44, and epithelial-specific antigen (ESA) (12). The expression of 
A

$\mathrm{Oh}$

$48 \mathrm{~h}$
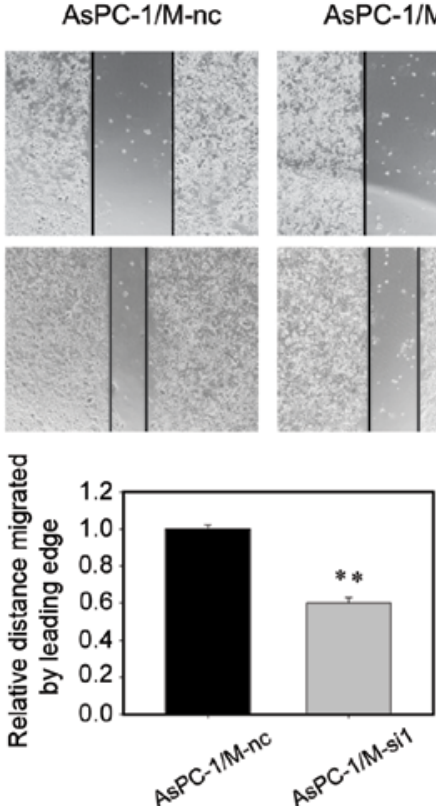

AsPC-1/M-si1
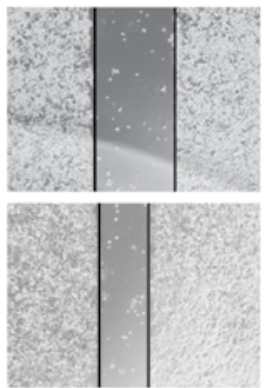

$48 \mathrm{~h}$
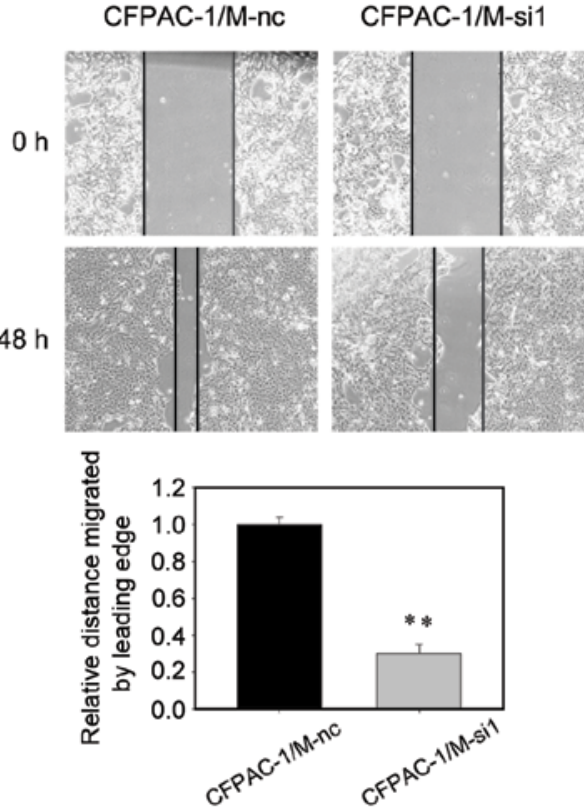

B
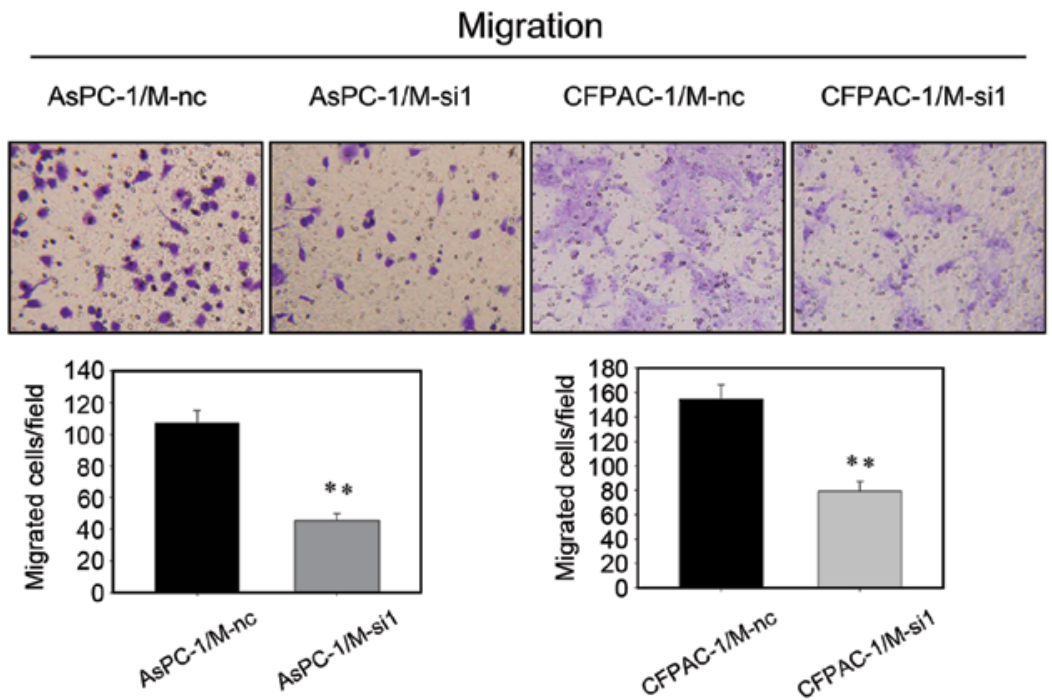

C

Invasion
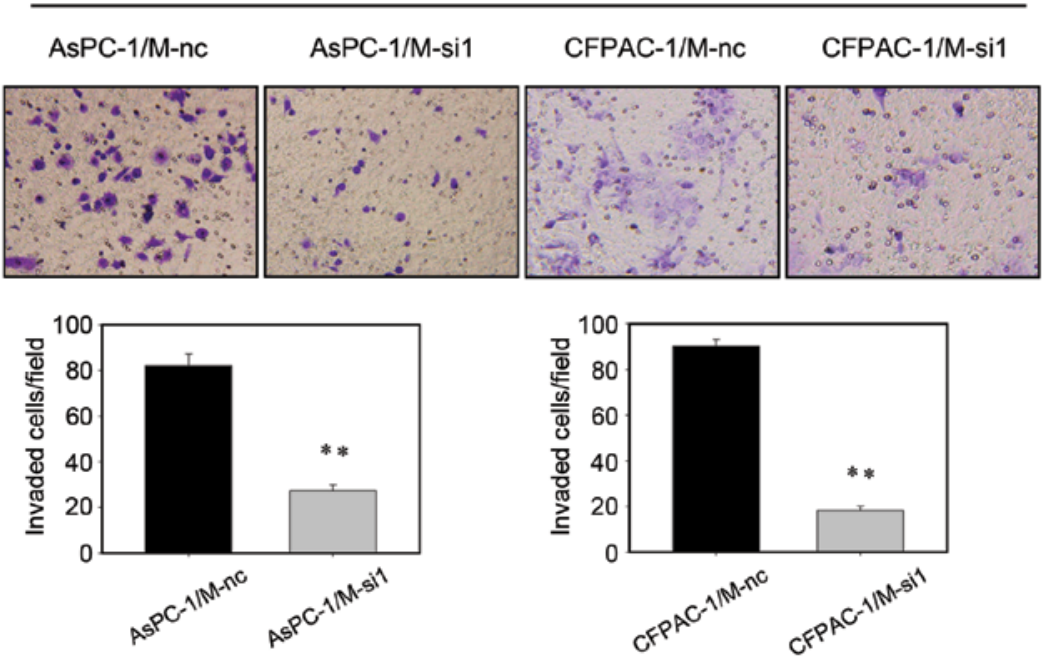

Figure 4. Metastasis-associated lung adenocarcinoma transcript-1 (MALAT-1) knockdown suppresses cell migration and invasion in vitro. (A) Cell mobility was investigated by wound healing assay. Migration and invasion ability was analyzed by (B) Transwell assay and (C) Boyden chamber, respectively (x200). Data are representative of at least three separate experiments. ${ }^{* *} \mathrm{P}<0.01$ compared with the respective control groups. 
A
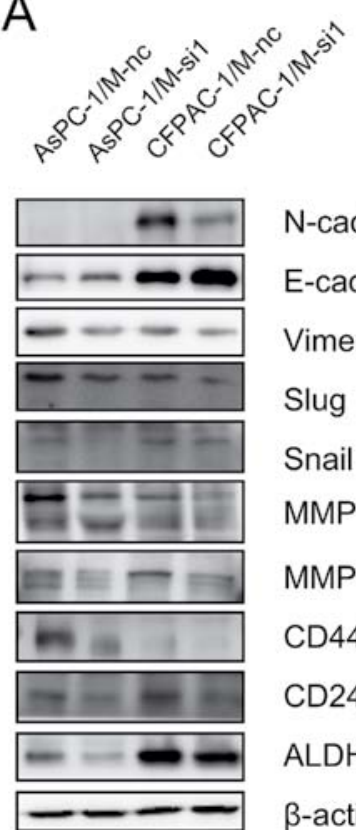

Slug

Snail

MMP-2

MMP-9

CD44

CD24

ALDH

$\beta$-actin

$\mathrm{N}$-cadherin

E-cadherin

Vimentin

B

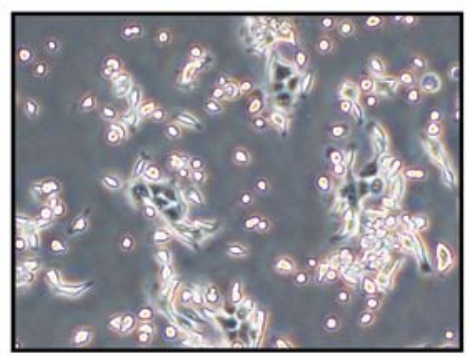

CFPAC-1/M-nc

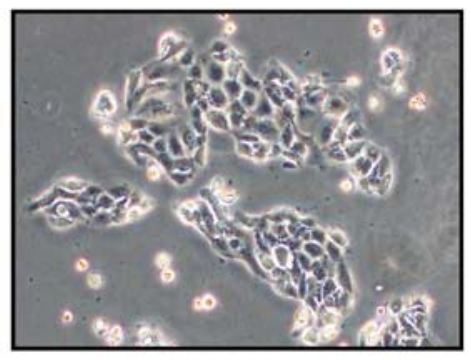

CFPAC-1/M-si1

Figure 5. Metastasis-associated lung adenocarcinoma transcript-1 (MALAT-1) regulates epithelial-mesenchymal transition (EMT)-associated genes and cancer stem-like cell marker expression. (A) MALAT-1 regulates the expression of EMT-marker genes including Snail, Slug, N-cadherin, vimentin, E-cadherin, matrix metalloproteinase-2 (MMP-2) and MMP-9, and cancer stem-like cell marker genes such as CD44, CD24 and aldehyde dehydrogenase (ALDH). (B) Suppression of MALAT-1 expression led to morphological changes in CFPAC-1 cells. Data are representative of at least three separate experiments.

aldehyde dehydrogenase (ALDH) was also used as special markers of pancreatic CSCs (13). In order to gain insight into the role of MALAT-1 involved in regulating pancreatic CSCs, we performed western blot analysis to analyze the altered expression of pancreatic CSC-associated markers following MALAT-1 downregulation, and the results showed that the levels of CD44,CD24 and ALDH were significantly decreased in M-sil compared with the M-nc groups (Fig. 5A). The above data suggest that MALAT-1 facilitates pancreatic cancer cell progression possibly through acquiring cancer stem-like properties.

\section{Discussion}

LncRNAs are broadly defined as transcribed RNA molecules with a length greater than 200 nucleotides and lacking an open reading frame of significant length (less than 100 amino acids). Studies have begun to provide insight into the critical roles played by lncRNA in a variety of cellular processes, including differentiation, development and tumorigenesis (14). The importance of lncRNAs had opened a new field of focus for cancer research.

MALAT-1, also known as nuclear enriched transcript-2 (NEAT-2), is a widely expressed IncRNA implicated in regulation of alternative splicing or gene expression. Emerging evidence indicates that MALAT-1 is overexpressed in many solid tumors, such as hepatocellular carcinoma (15), gastric (16) and gallbladder cancer (17), and it plays a significant role in the molecular events of neoplasia (4). Considering the observation that MALAT-1 is implicated in multiple aspects of tumor biological processes, we investigated the role of MALAT-1 in pancreatic cancer. In the present study, we first compared the level of MALAT-1 expression between six cases of fresh tumor tissue samples and adjacent non-cancerous pancreas tissues, and the results revealed higher MALAT-1 level in pancreatic cancer tissues. Furthermore, all seven pancreatic cancer cell lines had relatively higher level of MALAT-1 as compared to HPDE. A recent study showed that MALAT-1 expression level correlated with tumor size, tumor stage and depth of invasion in pancreatic cancer formalin-fixed, paraffin embedded tissues (18). The above results indicate that MALAT-1 overexpression may play an important role in pancreatic cancer.

Next, to specifically determine the contributions of MALAT-1 in the regulation of pancreatic cancer, we modulated its expression in AsPC-1 and CFPAC-1 cell lines. The cell growth results revealed that stably decreased expression of MALAT-1 could lead to reduced cell proliferation and colony formation. Furthermore, flow cytometry analyses and western blot results showed that suppression of MALAT-1 expression could cause $\mathrm{G}_{2} / \mathrm{M}$ cell cycle arrest and induce cell apoptosis. These results suggested an inhibiting effect of MALAT-1 downregulation on pancreatic cancer cell growth through inducing $\mathrm{G}_{2} / \mathrm{M}$ cell cycle arrest and promoting cell apoptosis.

Moreover, wound healing assay, Transwell assays and Boyden chamber revealed that silencing MALAT-1 could clearly inhibit pancreatic cancer cell migratory and invasive ability in vitro. Recent evidence suggests that EMT of pancreatic cancer cells contributes to the development and increase in invasiveness and metastasis (9). During EMT, epithelial cells undergo profound phenotypic changes such as loss of cell-cell adhesion, loss of cell polarity and acquisition of migratory and invasive properties. It should be noted that EMT is characterized by a downregulation of epithelial markers and an upregulation of mesenchymal markers and EMT-related transcriptional factors. In addition, relative research demonstrated that MMPs play a key role in aberrant pancreatic cell growth 
and tumor formation, since they provide space for the tumor to grow and release various growth factors that drive tumor proliferation and progression (19). In our studies, we found that suppressing MALAT-1 expression decreased MMP-2, MMP-9 and EMT-marker genes including Snail, Slug, N-cadherin, and vimentin, while it upregulated the expression of E-cadherin significantly. In addition, morphological change was observed in CFPAC-1 after MALAT-1 downregulation.

Researchers recently demonstrated that the presence of CSCs in pancreatic tumors contributes to the early metastasis and chemotherapeutic drug resistance of pancreatic cancer (20). Accumulating evidence suggests that EMT is important in cancer progression conceivably by inducing stem cell properties to cancer cells $(21,22)$. Therefore, we speculated that MALAT-1 induced EMT and acquired stem-like properties to facilitate tumor invasion and metastasis. The protein expression of pancreatic CSC markers including CD44, CD24 and ALDH were compared between M-nc and M-sil groups. As expected, the results showed that levels of all these markers decreased after MALAT-1 knockdown. Taken together, these results indicated that MALAT-1 facilitated pancreatic cancer progression probably through inducing EMT and acquiring cancer stem-like properties. However, the detailed molecular mechanisms of MALAT-1 regulating pancreatic CSCs and tumor progression require further exploration.

In conclusion, our findings reveal strong expression of MALAT-1 in patients with pancreatic cancer, and suggest that MALAT-1 may serve as an oncogenic lncRNA that promotes pancreatic cancer cell growth and progression. Since MALAT-1 is associated with the malignancy phenotypes of pancreatic cancer, further study is required to determine the potential roles of MALAT- 1 as a candidate therapeutic target in the clinic.

\section{Acknowledgements}

This study was supported by the National Natural Science Foundation of China (grant nos. 81101846, 81171887, 91229117 and 31101016), Program of Shanghai Subject Chief Scientist (grant no. 12XD1404200), Shanghai International Science and Technology Cooperation Project (grant no. 12410709000) and Shanghai Science and Technology Committee (grant no. 11DZ1922002).

\section{References}

1. Jemal A, Bray F, Center MM, Ferlay J, Ward E and Forman D: Global cancer statistics. CA Cancer J Clin 61: 69-90, 2011.

2. He Y, Meng XM, Huang C, Wu BM, Zhang L, Lv XW and Li J: Long noncoding RNAs: novel insights into hepatocelluar carcinoma. Cancer Lett 344: 20-27, 2014.

3. Ji P, Diederichs S, Wang W, Böing S, Metzger R, Schneider PM, Tidow N, Brandt B, Buerger H, Bulk E, Thomas M, Berdel WE, Serve H and Müller-Tidow C: MALAT-1, a novel noncoding RNA, and thymosin beta4 predict metastasis and survival in early-stage non-small cell lung cancer. Oncogene 22: 8031-8041, 2003.
4. Gutschner T, Hämmerle M and Diederichs S: MALAT1 - a paradigm for long noncoding RNA function in cancer. J Mol Med (Berl) 91: 791-801, 2013.

5. Thon JN, Devine MT, Jurak Begonja A, Tibbitts J and Italiano JE Jr: High-content live-cell imaging assay used to establish mechanism of trastuzumab emtansine (T-DM1)mediated inhibition of platelet production. Blood 120: 1975-1984, 2012.

6. Li W, Xie L, Chen Z, Zhu Y, Sun Y, Miao Y, Xu Z and Han X: Cantharidin, a potent and selective PP2A inhibitor, induces an oxidative stress-independent growth inhibition of pancreatic cancer cells through $\mathrm{G}_{2} / \mathrm{M}$ cell-cycle arrest and apoptosis. Cancer Sci 101: 1226-1233, 2010.

7. Stark GR and Taylor WR: Control of the $\mathrm{G}_{2} / \mathrm{M}$ transition. Mol Biotechnol 32: 227-248, 2006.

8. Ayyagari VN and Brard L: Bithionol inhibits ovarian cancer cell growth in vitro - studies on mechanism(s) of action. BMC Cancer 14: 61, 2014.

9. Pan JJ and Yang MH: The role of epithelial-mesenchymal transition in pancreatic cancer. J Gastrointest Oncol 2: 151-156, 2011.

10. Min C, Eddy SF, Sherr DH and Sonenshein GE: NF-kappaB and epithelial to mesenchymal transition of cancer. J Cell Biochem 104: 733-744, 2008.

11. Mani SA, Guo W, Liao MJ, Eaton EN, Ayyanan A, Zhou AY, Brooks M, Reinhard F, Zhang CC, Shipitsin M, Campbell LL, Polyak K, Brisken C, Yang J and Weinberg RA: The epithelialmesenchymal transition generates cells with properties of stem cells. Cell 133: 704-715, 2008.

12. Lee CJ, Dosch J and Simeone DM: Pancreatic cancer stem cells. J Clin Oncol 26: 2806-2812, 2008.

13. Rasheed ZA, Yang J, Wang Q, Kowalski J, Freed I, Murter C, Hong SM, Koorstra JB, Rajeshkumar NV, He X, Goggins M, Iacobuzio-Donahue C, Berman DM, Laheru D, Jimeno A, Hidalgo M, Maitra A and Matsui W: Prognostic significance of tumorigenic cells with mesenchymal features in pancreatic adenocarcinoma. J Natl Cancer Inst 102: 340-351, 2010.

14. Maruyama R and Suzuki H: Long noncoding RNA involvement in cancer. BMB Rep 45: 604-611, 2012.

15. Lai MC, Yang Z, Zhou L, Zhu QQ, Xie HY, Zhang F, Wu LM, Chen LM and Zheng SS: Long non-coding RNA MALAT-1 overexpression predicts tumor recurrence of hepatocellular carcinoma after liver transplantation. Med Oncol 29: 1810-1816, 2012.

16. Wang J, Su L, Chen X, Li P, Cai Q, Yu B, Liu B, Wu W and Zhu Z: MALAT1 promotes cell proliferation in gastric cancer by recruiting SF2/ASF. Biomed Pharmacother 68: 557-564, 2014.

17. Wu XS, Wang XA, Wu WG, Hu YP, Li ML, Ding Q, Weng H, Shu YJ, Liu TY, Jiang L, Cao Y, Bao RF, Mu JS, Tan ZJ, Tao F and Liu YB: MALAT1 promotes the proliferation and metastasis of gallbladder cancer cells by activating the ERK/MAPK pathway. Cancer Biol Ther 15: 806-814, 2014.

18. Liu JH, Chen G, Dang YW, Li CJ and Luo DZ: Expression and prognostic significance of lncRNA MALAT1 in pancreatic cancer tissues. Asian Pac J Cancer Prev 15: 2971-2977, 2014.

19. Łukaszewicz M, Mroczko B and Szmitkowski M: The role of metalloproteinases and their inhibitors in pancreatic cancer. Postepy Hig Med Dosw (Online) 62: 141-147, 2008 (In Polish).

20. Li Y, Kong D, Ahmad A, Bao B and Sarkar FH: Pancreatic cancer stem cells: emerging target for designing novel therapy. Cancer Lett 338: 94-100, 2013.

21. Sarkar FH, Li Y, Wang Z and Kong D: Pancreatic cancer stem cells and EMT in drug resistance and metastasis. Minerva Chir 64: 489-500, 2009.

22. Thiery JP, Acloque H, Huang RY and Nieto MA: Epithelialmesenchymal transitions in development and disease. Cell 139: 871-890, 2009. 